\title{
Slide-ring gel: Topological gel with freely movable cross-links
}

\author{
Takeshi Karino $^{\mathrm{a}, 1}$ Mitsuhiro Shibayama ${ }^{\mathrm{a}, 1}$ Kohzo Ito ${ }^{\mathrm{b}, 1, *}$ \\ ${ }^{a}$ Neutron Science Laboratory, Institute for Solid State Physics, University of Tokyo, Tokai, Ibaraki, 319-1106, Japan \\ ${ }^{\mathrm{b}}$ Graduate School of Frontier Sciences, University of Tokyo, Kashiwa, Chiba 277-8561, Japan
}

\begin{abstract}
The structure of "slide-ring (SR) gel" was investigated by means of small angle neutron scattering (SANS). The SR gel was synthesized by coupling of $\alpha$-cyclodextrin molecules on polyrotaxane chains. A cross-linking point of the SR gel has a shape of "figure-of-eight". Two dimensional SANS patterns for uniaxially-stretched SR gels showed a "normal" butterfly pattern. This result indicates that the cross-links slide along the polymer chain so as to minimize the local strain as a "pulley". The pulley effect was found to be significant to reduce spatial inhomogeneities inherent in polymer gels.
\end{abstract}

Key words: slide-ring gel, pulley effect, figure-of eight cross-linking

\section{Introduction}

Polymer gels are three-dimensional polymer networks consisting of polymer chains, cross-links, and large amount of solvent. In general, cross-links were introduced by physical or chemical bonding. Hence, the concentration fluctuations in polymer gels are topologically frozen by nature due to the presence of permanent networks. These types of concentration fluctuations are called spatial inhomogeneities. The spatial inhomogeneities in polymer gel can be observed by scattering methods, such as an excess scattering in small angle neutron scattering (SANS) and a speckle pattern in light scattering[1]. It is preferable to reduce the spatial inhomogeneities from application points of view since spatial inhomogeneities degrade transparency, mechanical strength, and deformability. In order to circumvent these problems, novel types of

\footnotetext{
* Graduate school of Frontier Sciences, University of Tokyo, 5-1-5 Kashiwanoha, Kashiwa, Chiba 277-8581 JAPAN. Phone: +81 4-7136-3756 Fax: +81 4-7134-6069, Email: kohzo@k.utokyo.ac.jp

1 This work was supported by Core Research for Evolutional Science and Technology (CREST), Japan Science and Technology Agency, Japan.
}

gels have recently been synthesized, such as a doublenetwork gel[2] and a nano-composite gel[3].

We have developed a new kind of gel called "slidering (SR) gel" or "topological gel"[4]. The main components of the SR gel are polyrotaxane comprising poly(ethylene glycol) and $\alpha$-cyclodextrin. These polyrotaxane chains were cross-linked by coupling the $\alpha$-cyclodextrin rings. Fig. 1 shows the schematic illustration of the SR gel. As shown in the figure, a cross-link of SR gel can move freely along the polymer chain to equalize the tension of the polymer chains just like a pulley. An essential difference between the SR gels and conventional polymer gel is the presence of movable cross-links. It is naively expected that nanoscopic spatial inhomogeneities and local stresses may be automatically relaxed by a sliding motion of the movable cross-links. As a matter of fact, it is confirmed that these movable cross-links allow high extensibility, large degree of swelling, and large reversible-deformability[4]. Although the concept of movable cross-links itself was proposed theoretically by Edwards in 1980s[5] and was recently revisited by Rubinstein and Panyukov[6], the success of the preparation of SR gels opened a new field of material sciences as was addressed by Granick and Rubinstein[7].

The sliding motion of $\alpha$-cyclodextrin molecule on 


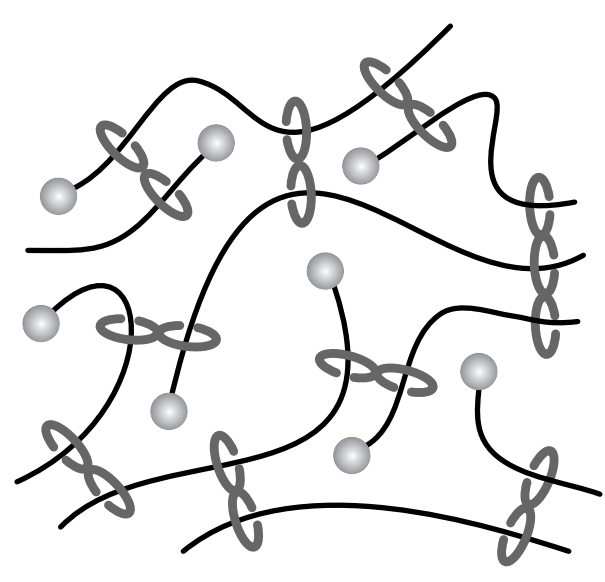

Fig. 1. Schematic illustration of Slide-ring gel.

polyrotaxane chain was confirmed by dynamic light scattering (DLS)[8]. The decay-rate distribution function of polyrotaxane in $1 \mathrm{~N} \mathrm{NaOHaq}$ had three peaks. In general, the decay-rate distribution function of polymer solutions show a fast mode related to cooperativediffusion mode and a slow mode related to self-diffusion mode. In the decay-rate distribution function of polyrotaxane chain, a new mode appeared between the fast and slow modes. In order to assign the newlyobserved mode, DLS measurements were also carried out on poly(ethylene glycol) and $\alpha$-cyclodextrin solutions with the same condition. The fast and slow modes of polyrotaxane correspond to those of poly(ethylene glycol). Therefore, the new mode was assigned to the sliding motion of $\alpha$-cyclodextrin molecule on polyrotaxane.

The spatial inhomogeneities in a gel can be amplified by stretching. The so-called butterfly pattern with a two-lobe isointensity scattering pattern appears. A butterfly pattern is observed not only in polymer gels but also in polymer solutions under a flow field and in polymer films under uniaxial stretching. Scattering theories predict a prolate pattern elongated in the direction perpendicular to the stretching direction of polymer chains. Such a butterfly pattern is called a "normal butterfly pattern". On the other hand, a scattering pattern for a stretched polymer gel is prolate in the direction to the stretching direction. This type of pattern is called an "abnormal butterfly pattern". Abnormal butterfly patterns in polymer gels were experimentally reported by Mendes[9] and Ramzi[10] for polystyrene and by Shibayama et al.[11] for thermosensitive weakly charged gels. These results concluded that an appearance of abnormal butterfly pattern was ascribed to an increase of spatial inhomogeneities predominantly in the stretching direction. These abnormal patterns are explained theoretically by Onuki[12], Rabin et al.[13], and Panyukov and Rabin[14]. According to these theories, the scattering function is de- scribed by a sum of a Lorentz type scattering function (equivalent to an Ornstein-Zernike function) representing thermal fluctuations and a squared-Lorentz type function corresponding to the cross-links inhomogeneities. Hence, in the absence of spatial inhomogeneities, the butterfly pattern is reduced to a normal butterfly pattern. Therefore, analysis of a butterfly pattern is a key to understand the spatial inhomogeneities of gels.

In this paper, we focus on the butterfly patterns in stretched SR gels obtained by SANS and discuss the relationship between the inhomogeneities and sliding motion.

\section{Experimental}

Since the details of the preparation procedure of polyrotaxanes and SR gels is reported elsewhere[15][16], a brief recipe is given below. $0.9 \mathrm{~g}$ of poly(ethylene glycol) (PEG) and $3.6 \mathrm{~g}$ of $\alpha$ cyclodextrin (CD) were dissolved in $15 \mathrm{~g}$ of water at 80 ${ }^{\circ} \mathrm{C}$ and kept at $5{ }^{\circ} \mathrm{C}$ overnight to yield white paste. The molecular weight of PEG was $1.0 \times 10^{5}$. The polydispersity of PEG was 1.12. The paste was dried, followed by addition of an excess of 2,4-dinitrofluorobenzene together with dimethylformamide (DMF). The mixture was stirred in a nitrogen atmosphere at room temperature overnight and then was dissolved in DMSO and precipitated twice from a $0.1 \%$ sodium chloride aqueous solution to give a yellow product. The product was collected, washed with water and methanol, and dried to produce the polyrotaxane. The filling ratio was determined by ${ }^{1} \mathrm{H}$ NMR. The number of $\mathrm{CD}$ molecules on PEG chains was calculated from the peak height of CD and PEG. The filling ratio of CD was $17 \%$.

The SR gels were prepared in deuterated dimethyl sulfoxide (99.9\% deuteration) (d-DMSO), where N,N'carbonyldiimidazole was used as cross-linker. The concentration of the polyrotaxane was $10 \mathrm{wt} \%$. The crosslinker concentrations, $C_{\mathrm{X}}$ 's were chosen to be $0.7,1.0$, $1.5 \mathrm{wt} \%$. Accordingly, the sample codes were defined as CX07, CX10, CX15.

Small-angle neutron scattering (SANS) experiments were carried out at the SANS-U spectrometer at Institute for Solid State Physics, University of Tokyo, Tokai, Japan. Two-dimensional scattering intensity patterns were collected with a two-dimensional detector $(64 \times$ $64 \mathrm{~cm}^{2}$ ) placed at $8 \mathrm{~m}$ position from the samples. The incident neutron wavelength was $7.0 \AA$. The observed scattering intensity functions were corrected for air scattering, incoherent scattering, and transmission and then were rescaled to the absolute intensity, $I(q)$, with a polyethylene (Lupolen) secondary standard. 


\section{Results and Discission}

Polyrotaxane is soluble exclusively in DMSO and $1 \mathrm{~N}$ $\mathrm{NaOH}$ aq. CD molecules form a cluster due to hydrogen bonding between $\mathrm{OH}$ groups on $\mathrm{CD}$ molecules. This is why the solubility of polyrotaxane decreases. The DLS results indicated that a formation of CD cluster in DMSO was larger than that in $1 \mathrm{~N} \mathrm{NaODaq.} \mathrm{Since}$ the hydroxyl groups on CD molecule are dissociated in a strong alkali solution, CD molecules on a PEG chain are dispersed from each other due to repulsive interaction. On the other hand, DMSO hardly prevents CD molecules from forming hydrogen bonding clusters. This aggregation behavior of CD molecules can be monitored by SANS. It was found that the polyrotaxane chains in d-DMSO takes a rodlike conformation due to a stacking of CD molecules on PEG chain, whereas a Gaussian chain conformation is preferred in $\mathrm{NaOD} a q$ because of electrostatic repulsion between dissociated CD molecules.[16] The scattering function of a SR gel in NaOD aq showed a minimum around the sol-gel transition and is described by Ornstein-Zernike function. On the other hand, the scattering function of a SR gel in d-DMSO increased monotonically with increasing the cross-linker concentration and is given by the sum of a squared-Lorentz function and a scattering function for a rod. Therefore, by studying the scattering intensity functions, one can deduce the efficiency of the pulley effect since the pulley effect is strongly related to the stacking behavior of CD molecules on PEG chains[16].

Fig. 2 shows the cross-link concentration dependence of the scattering intensity, $I(q)$. In general, $I(q)$ for polymer gels increases with increasing the cross-link density. This is due to an increase of frozen inhomogeneities introduced by cross-linking. However, interestingly enough, $I(q)$ of the SR gels decreased with increasing $C_{\mathrm{X}}$. These results suggest that the concentration fluctuations of the SR gel are suppressed by an introduction of cross-links. According to the Onuki's theory[12], the zero- $q$ scattering intensity is given by,

$I(q=0) \approx \frac{k T \phi^{2}}{M_{\mathrm{OS}}}\left(1+p_{\mathrm{int}} \frac{\mu}{M_{\mathrm{OS}}}\right)$

where $\phi$ is the polymer volume fraction, $M_{\mathrm{OS}}$ is bulk modulus, $\mu$ is the shear modulus, and $p_{\text {int }}$ is the degree of inhomogeneities. Both $M_{\mathrm{OS}}$ and $p_{\text {int }}$ increase with increasing $C \mathrm{x}$. In the case of conventional chemical gels, $I(q)$ increases with increasing $C \mathrm{x}$ because an increase of $p_{\text {int }}$ is larger than that of $M_{\mathrm{OS}}$. On the other hand, in the case of the SR gel, the variations of $p_{\text {int }}$ are suppressed by the pulley effect although $M_{\mathrm{OS}}$ is expected to increase as well as covalently bonded chemical gels. As a result, an decrease in $I(q)$ of SR gel was observed.

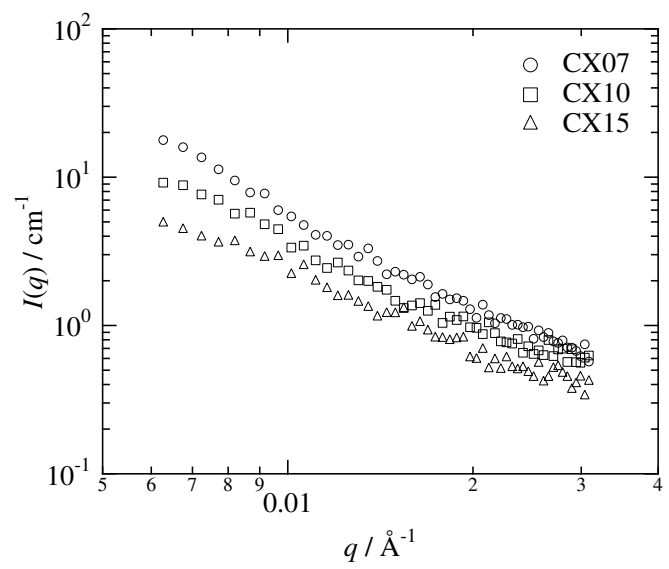

Fig. 2. Cross-link concentration dependence of scattering intensity for the SR gels.

Fig. 3 shows the two-dimensional SANS isointensity patterns of SR gels (CX10). The stretching direction was horizontal. $\lambda\left(=l / l_{0}\right)$ is the stretching ratio, and $l_{0}$ and $l$ are the sample lengths before and after stretching, respectively. As clearly shown, the scattering patterns at $\lambda=1.0$ are isotropic. On the other hand, the scattering patterns at $\lambda=4.0$ become elliptic perpendicular to the stretching direction. This type of scattering pattern is called a normal butterfly pattern. This is the first time to our knowledge to observe a normal butterfly pattern in a polymer gel. Conventional chemical gels show abnormal butterfly patterns. The origin of the appearance of abnormal butterfly patterns is explained by an amplification of inhomogeneities preferentially to the stretching direction. In the case of conventional gel, the spatial inhomogeneities become explicit by deformation since the polymer chains are connected by covalent or physical cross-links. On the other hand, the SR gels show normal butterfly patterns as shown in the figure. These results indicate that an increase of the spatial inhomogeneities by deformation is effectively suppressed by the pulley effect of the SR gels[17].

Fig. 4 shows the sector-averaged scattering intensities of the butterfly pattern shown in Fig. 3. The sector angle was $10^{\circ}$ in both the parallel and perpendicular directions. The scattering intensity in the parallel direction decreased by stretching. On the other hand, the scattering intensity in the perpendicular direction remains more or less invariant. The decrease in the scattering intensity in the parallel direction indicates that the spatial inhomogeneities was reduced by sliding cross-links. In the previous study[16], the scattering functions of a SR gel in d-DMSO were well described with a sum of a squared-Lorentz and a rod function given by 

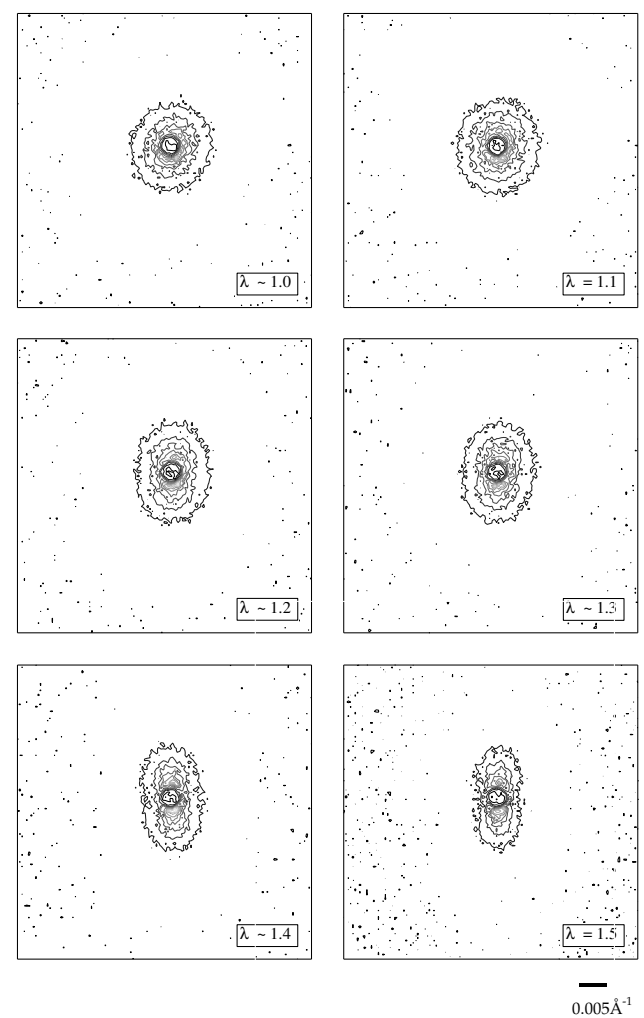

Fig. 3. Two-dimensional SANS isointensity patterns of SR gels (CX10).The stretching direction is horizontal.

$I(q)=\frac{I_{\mathrm{SL}}(0)}{\left(1+\Xi^{2} q^{2}\right)^{2}}+I(0)_{\mathrm{R}} q^{-1}$

where $I_{\mathrm{SL}}(0)$ and $I_{\mathrm{R}}(0)$ are the forward scattering intensities of the squared-Lorentz and the rod, respectively. $\Xi$ is the characteristic length of gel inhomogeneities. The first term in Eq. (2) shows the spatial inhomogeneities of the gel. The second term describes a rod-like chain behavior in the short-distance scale due to the stacking of CD molecules. The dashed lines in Fig. 4 are the fitted curves using Eq. (2). The fitting results agree well with the observed scattering curves. The $\Xi$ 's in the perpendicular direction are $155 \AA$ at both $\lambda=1.0$ and $\lambda=1.5$. On the other hand, $\Xi$ 's in the parallel direction are $144 \AA$ and $120 \AA$ at $\lambda=1.0$ and $\lambda=1.5$, respectively. This $\lambda$ dependence of $\Xi$ is completely opposite to that reported by Mendes et al[18] for conventional chemical gels. This means that the stretching mechanism of SR gels is different from that of conventional gels.

We propose a deformation mechanism of the SR gels on the basis of the experimental results. Fig. 5 shows the illustration of deformation mechanism of polymer gel. At undeformed state, cross-links are distributed

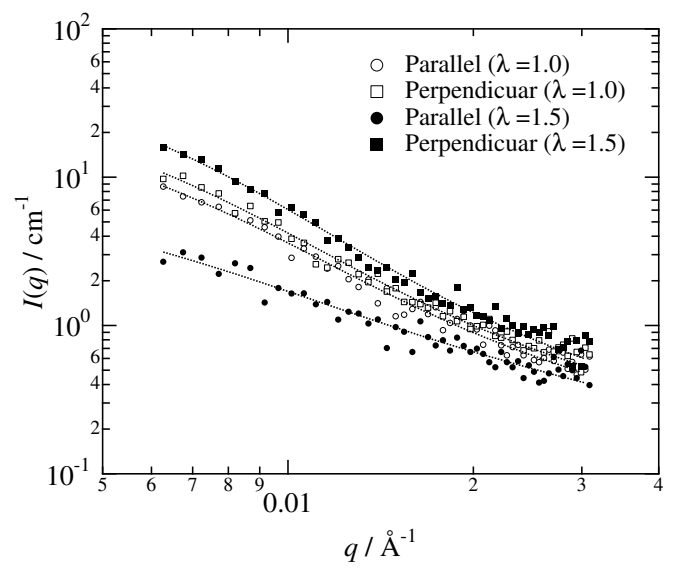

Fig. 4. Sector-averaged scattering intensities of the SR gel (CX10) in the parallel and perpendicular directions at $\lambda=1.0$ and $\lambda=1.5$.

in the polymer networks. By stretching, the polymer chains of conventional chemical gels are aligned in the stretching direction. On the other hand, the cross-links inhomogeneities are displaced along the stretching direction. This is why an abnormal butterfly pattern was observed. In the case of the SR gels, the spatial inhomogeneities do not increase in the stretching direction due to the presence of movable cross-links. By stretching, the SR gel tends to maintain its uniformity by sliding the cross-links along the polymer chain. Therefore, a normal butterfly pattern was observed.

\section{Conclusion}

The SR gel is a novel gel having movable cross-links along a polymer chain. This specific cross-linking structure of SR gel shows different mechanical properties in contrast to conventional chemical gels and rubbers. The new mode obtained by dynamic light scattering was assigned to the sliding mode of $\alpha$-cyclodextrin on poly(ethylene glycol). We carried out small angle neutron scattering (SANS) experiments in order to confirm the sliding motion of slide-ring (SR) gels under uniaxial stretching. The scattering intensity decreased with increasing the cross-link concentrations, $C_{\mathrm{X}}$ which is opposite to the case of conventional chemical gels. This implies that the spatial inhomogeneities decrease with $C_{\mathrm{X}}$. Under uniaxial stretching, the two-dimensional iso-intensity curve shows a normal butterfly pattern in which the scattering intensity increases in the perpendicular direction. This normal butterfly pattern was observed for the first time in polymer gels. By stretching, the scattering intensity in the parallel direction decreased with increasing the stretching ratio. The spatial inhomogeneities of the SR gel were reduced owing 
(a)

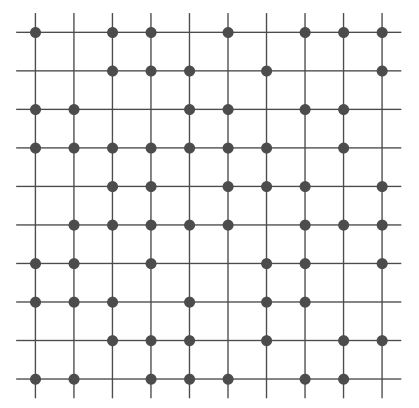

(b)

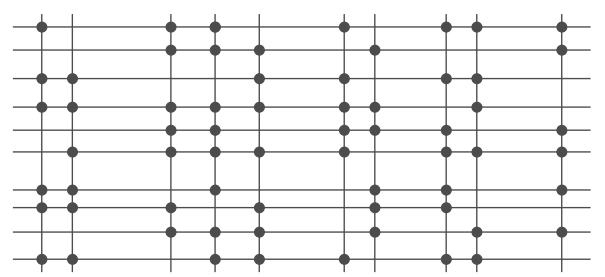

(c)

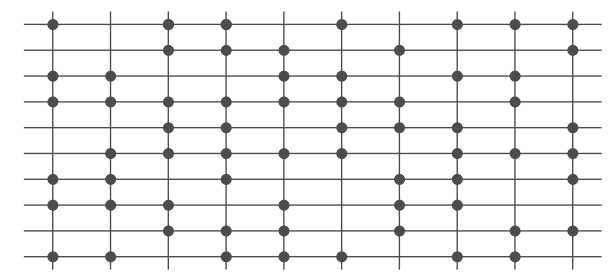

Fig. 5. At undeformed state (a), cross-links are randomly distributed in gel networks. By uniaxial deformation, cross-links of conventional chemical gel align to the stretching direction (b). On the other hand, cross-links of slide-ring gel distribute homogeneously (c). The stretching direction is horizontal.

to that the cross-links of the SR gels might move freely along the polymer chain. These results strongly support the pulley effect in the SR gels.

\section{Acknowledgment}

This work is supported by Core Research for Evolutional Science and Technology (CREST), Japan Science and Technology Agency, Japan.This work was also partially supported by the Ministry of Education, Science, Sports and Culture, Japan (Grant-inAid, 14045216 and 16350120)). The SANS experiment was performed with the approval of Institute for Solid State Physics, The University of Tokyo (Proposal Nos. 02- 2529, 03-3510), at Japan Atomic Energy Research Institute, Tokai, Japan.

\section{References}

[1] M. Shibayama, Macromol. Chem. Phys. 199 (1998) 1.
[2] Y. H. Na, T. Kurokawa, Y. Katsuyama, H. Tsukeshiba, G. P. Gong, Y. Osada, S. Okabe, T. Karino, M. Shibayama, Macromolecules, 37 (2004) 5370.

[3] K. Haraguchi, T. Takeshita, Adv. Mater. 14 (2002) 1121.

[4] Y. Okumura, K. Ito, Adv. Mater. 13 (2001) 485.

[5] S. F. Edwards, Th. Vilgis, Polymer 27 (1986) 483.

[6] M. Rubinstein, S. Panyukov, Macromolecules 35 (2002) 6670 .

[7] S. Granick, M. Rubinstein, Nature Mater. 3 (2004) 586.

[8] C. Zhao, Y. Domon, Y. Okumura, S. Okabe, M, Shibayama, K. Ito, J. Phys. : Condens. Matter 17 (2005) S2841.

[9] E. J. Mendes, P. Lindner, M. Buzier, F. Boue, J. Bastide, Phys. Rev. Lett. 66 (1991) 1595.

[10] A. Ramzi, A. Hakiki, J. Bastide, F. Boue, Macromolecules 30 (1997) 2963.

[11] M. Shibayama, K. Kawakubo, F. Ikkai, M. Imai, Macromolecules 31 (1998) 2586.

[12] A. Onuki, J. Phys. II 2 (1992) 45.

[13] Y. Rabin, R. Bruinsma, Europhys. Lett. 20 (1992) 79.

[14] S. Panyukov, Y. Rabin, Phys. Rep. 269 (1996) 1.

[15] J. Araki, C. Zhao, K. Ito, Macromolecules in press.

[16] T. Karino, Y. Okumura, K. Ito, M. Shibayama, Macromolecules 37 (2004) 6177.

[17] T. Karino, Y. Okumura, C. Zhao, T. Kataoka, K. Ito, M. Shibayama, Macromolecules 38 (2005) 6161.

[18] E. J. Mendes, R. Oeser, C. Hayes, F. Boue, J. Bastide, Macromolecules 29 (1996) 5574. 